\title{
Surgery combined with local 5-aminolevulinic acid-photodynamic therapy on skin cancer and its effect on the expression of cyclophilin A, cyclophilin B and CD147
}

\author{
LING GUO* and YINGSHENG HAN* \\ Department of Dermatology, Liaocheng People's Hospital, Liaocheng, Shandong 252000, P.R. China
}

Received February 17, 2017; Accepted May 18, 2017

DOI: $10.3892 / \mathrm{ol} .2017 .6275$

\begin{abstract}
The study evaluated an approach to treat skin cancer using surgery combined with local 5-aminolevulinic acid-photodynamic therapy (ALA-PDT). Seventy-six patients with skin cancer who were admitted to the Liaocheng People's Hospital from May 2014 to April 2015 were randomly divided into a control and an observation group (38 cases in each). The patients in the control group were treated with ALA-PDT alone. Those in the observation group were first subjected to surgical treatment, and then treated with ALA-PDT. The treatment efficacies of the two groups were compared. The expression of cancer markers CyPA, CyPB and CD147 were detected by immunohistochemical methods before and after the treatment. Our results showed the average healing time of the wounds of patients in the observation group was shorter, the number of treatments needed was less, the efficacy rate and the lesion appearance satisfaction were significantly higher, and the recurrence rate at 12 months after treatment and the incidence of adverse reactions were both significantly lower. Additionally, the levels of CyPA, CyPB and CD147 were reduced to a significantly higher degree after treatment in the observation group. No difference was found in the recurrence rate between the two groups at 6 months after treatment. We conclude that surgery combined with ALA-PDT is a safe and reliable treatment method, which can increase the survival rate while improving the recovery rate and appearance satisfaction in patients with skin cancer.
\end{abstract}

\section{Introduction}

As populations become increasingly aged and the magnitude of environmental pollution rises, the incidence of skin cancer also increases. Skin cancer is one of the most common

Correspondence to: $\mathrm{Dr}$ Yingsheng Han, Department of Dermatology, Liaocheng People's Hospital 67 Dongchang West Road, Liaocheng, Shandong 252000, P.R. China

E-mail: h838nt@163.com

*Contributed equally

Key words: 5-aminolevulinic acid-photodynamic therapy, skin cancer, cyclophilin A, cyclophilin B, CD147 malignancies with high invasiveness and metastasis rates. It usually occurs in the exposed parts of the human body, such as the neck, face and upper arm. The most common types are basal cell and squamous cell carcinomas (1). Local invasion by skin cancer cells can cause damage to the local tissues, generating deformations that affect the normal appearance of the patient (2).

Many treatment methods, including traditional surgery, radiotherapy, chemotherapy, cryotherapy and photodynamic therapy can be used to treat skin cancers (3). Nevertheless, surgery remains as the mainstay of treatment. In addition, painless photodynamic therapy (PDT) with its excellent safety profile, fine cosmetic results and low recurrence rate has become a standard option for certain cases even though it is not cost-effective $(4,5)$. Compared with the traditional photodynamic therapy, 5-aminolevulinic acid-photodynamic therapy (ALA-PDT), the second-generation 5-aminolevulinic acid (5-ALA), is used as the photosensitizer. In this case, absorption by the target tissues occurs faster, prompt excretion from the body ensures minimal photosensitivity adverse effects, and a shorter time course of lightproof treatment is necessary. CyPA and CyPB are widely distributed proteins expressed in various types of human cells, may be involved in the apoptosis of skin cancer cells, and can be used as markers for progression of skin cancer.

In the present study, patients with skin cancer were treated with surgery and local ALA-PDT to evaluate the efficacy and safety of this method as compared to ALA-PDT alone, and the findings were reported.

\section{Materials and methods}

\section{Clinical data}

General information. Seventy-six patients with skin cancer who were admitted to the Liaocheng People's Hospital were selected from May 2014 to April 2015 and were randomly divided into a control or observation group (38 cases in each). The patients in the observation group were first subjected to surgical treatment, and then treated with ALA-PDT. The patients in the control group underwent ALA-PDT surgery alone. There were three main inclusion criteria: i) patients had the skin cancer diagnosis confirmed by histopathology; ii) they had not been treated with laser, freezing or topical treatments before the diagnosis and iii) the patients all signed informed 
Table I. General information for patients in the two groups.

\begin{tabular}{lcccr}
\hline Items & $\begin{array}{c}\text { Observation group } \\
(\mathrm{n}=38)\end{array}$ & $\begin{array}{c}\text { Control group } \\
(\mathrm{n}=38)\end{array}$ & $\mathrm{t} / \chi^{2}$ & P-value \\
\hline Age range (years) & $40-75$ & $40-72$ & & \\
Sex (male/female) & $20 / 18$ & $22 / 16$ & 0.213 & 0.645 \\
Mean age (years) & $57.56 \pm 6.47$ & $58.34 \pm 6.56$ & 0.522 & 0.603 \\
BMI (kg/m $\left.{ }^{2}\right)$ & $22.43 \pm 3.45$ & $22.56 \pm 3.38$ & 0.166 & 0.869 \\
Mean duration & $14.56 \pm 6.47$ & $15.34 \pm 6.56$ & 0.522 & 0.603 \\
of disease (months) & & & & \\
Skin lesion area (cm $\left.{ }^{2}\right)$ & $2.43 \pm 0.45$ & $2.56 \pm 0.48$ & 1.218 & 0.227 \\
Disease type (n, \%) & & & & 0.610 \\
$\quad$ Basal cell carcinoma & $21(55.26)$ & $24(63.16)$ & 0.260 & 0.610 \\
$\quad$ Squamous cell carcinoma & $17(44.74)$ & $14(36.84)$ & 0.260 & \\
\hline
\end{tabular}

consent. The exclusion criteria included patients with severe organ dysfunction, immune system diseases and eczema or fungal infections in the vicinity of the skin lesion area. No significant differences following a comparison og the general information between the two groups ( $P>0.05$, Table I). This study was approved by the Ethics Committee of Liaocheng People's Hospital. Signed written informed consents were obtained from all participants or their families before the study.

Treatment. The patients in the control group were only treated with ALA-PDT. Sodium chloride solution ( $0.9 \%)$ was used to clean the lesion and the surrounding skin, followed by cleaning with sterile cotton balls. The lesion was infiltrated with ozone solution for $30 \mathrm{~min}$. 5-ALA was dissolved in $1 \mathrm{ml}$ of a $5 \%$ ozone solution to produce $10 \%$ solution of 5-aminopentanoic acid. The $10 \%$ solution of 5-aminopentanoic acid was then applied to the lesion and the surrounding skin (approximately $0.5 \mathrm{~cm}$ beyond the lesion). The lesion area was coated with opaque material, which was removed $4 \mathrm{~h}$ later. The lesion area was then irradiated with photodynamic therapy apparatus (Guilin Xingda Optoelectronic Medical Instruments Co., Ltd., Guangxi, China) with an output power of $200 \mathrm{~mW} / \mathrm{cm}^{2}$, a wavelength of $635 \mathrm{~nm}$ and a light dose of $120 \mathrm{~J} / \mathrm{cm}^{2}$. The lesion area was treated for 40 min each time and once per week until the tumor was completely removed.

The patients in the observation group were treated with surgery and ALA-PDT. Patients were treated with $2 \%$ lidocaine for local anesthesia. An incision was located $2 \mathrm{~mm}$ beyond the edge of the lesion. The lesion was removed from the subcutaneous fat layer, fascia surface or deep skin and periosteal surface. Local bleeding was controlled. A direct suture or a suitable flap was selected according to the size and depth of the lesion to repair the wound. ALA-PDT was performed immediately after hemostasis applying the same method used for the control group.

Detection of indicators. Histopathological examination was performed before and after treatment, the specimen tissue was embedded with paraffin and placed at $-60^{\circ} \mathrm{C}$. The expression of CyPA, CyPB and CD147 was detected by immunohistochemical staining of specimens. The necessary reagents were provided by Beijing Zhongshan Jinqiao Biotechnology
Co. Ltd., (Guangdong, China). The tissue samples were sliced and dewaxed according to standard procedure. After washing, incubation and color development, the tissues were observed under a microscope. Pure water was used to wash the samples and terminate color development. Hematoxylin staining (30 sec) was applied twice and the samples were then sealed with neutral gum.

Follow-up. After treatment, the patients were followed up for 12 months. The efficacy of the treatment and patient satisfaction levels were recorded. The appearance of the skin at the lesion site and detected scar hyperplasias were recorded.

\section{Treatment efficacy and evaluation criteria}

Determination of treatment efficacy. Complete remission (CR) occurred when the pigmentation was significantly improved, the skin lesions completely disappeared, and there was no malignancy on pathology samples after treatment and no recurrences within 6 months. With partial remission (PR) the hyperpigmentation was lessened and the lesion area was reduced by $\geq 50 \%$ of the original. A failed treatment meant no improvement was seen in pigmentation, the skin lesions decreased by $<50 \%$ in size and the malignancy recurred within 6 months. The treatment efficiency was calculated by adding the percentages of CR and PR.

Observation of the surgical treatment outcomes in the two groups. The average times to wound healing and the number of treatments needed were recorded. The patient's satisfaction with the postoperative appearance was classified as one of three levels (very satisfied, generally satisfied and not satisfied). The percentage of patient satisfaction was calculated by adding the percentage of very satisfied and generally satisfied patients.

Observation of the incidence of adverse reactions in the two groups. The cases of burning, redness, swelling, pain and other adverse reactions were recorded. All the patients were followed up for 12 months after treatment. Any pathological changes of the lesion were examined. The recurrence rate was recorded at 6 and 12 months after treatment.

The expression levels of CyPA, CyPB and CD147 before and after treatment were detected by immunohistochemistry. Eight visual fields were randomly selected under high magnification (x400) and 100 cells were selected for each visual 
Table II. Comparison of the treatment efficiency between the two groups ( $\mathrm{n}, \%)$.

\begin{tabular}{lccccc}
\hline Groups & N (cases) & CR & PR & Invalid & Treatment efficiency \\
\hline Observation & 38 & $25(65.79)$ & $12(31.58)$ & $1(2.64)$ & $37(97.37)$ \\
Control & 38 & $13(34.21)$ & $16(42.11)$ & $9(23.69)$ & $29(76.32)$ \\
\hline
\end{tabular}

Treatment efficiencies of the two groups compared using rank sum test, $\mathrm{Z}=3.138, \mathrm{P}=0.002$. CR, complete remission; $\mathrm{PR}$, partial remission.

Table III. Comparison of the treatment outcomes between the two groups.

\begin{tabular}{lcccc}
\hline Groups & $\begin{array}{c}\mathrm{N} \\
\text { (cases) }\end{array}$ & $\begin{array}{c}\text { Average healing time } \\
\text { (days) }\end{array}$ & $\begin{array}{c}\text { Number of treatments } \\
\text { needed }\end{array}$ & $\begin{array}{c}\text { Patient satisfaction } \\
(\mathrm{n}, \%)\end{array}$ \\
\hline Observation & 38 & $13.32 \pm 3.23$ & $2.43 \pm 1.24$ & $36(94.73)$ \\
Control & 38 & $15.34 \pm 3.24$ & $3.45 \pm 1.38$ & $28(73.64)$ \\
$\mathrm{t} / \chi^{2}$ & & 2.722 & 3.389 & 4.862 \\
P-value & & 0.008 & 0.001 & 0.027 \\
\hline
\end{tabular}

Table IV. Comparison of the incidence of adverse reactions between the groups (n, \%).

\begin{tabular}{|c|c|c|c|c|c|}
\hline Groups & $\mathrm{N}$ (cases) & Burning sensation & Redness and swelling & Pain & Complication rate \\
\hline Observation & 38 & $1(2.63)$ & $0(0.00)$ & $1(2.63)$ & $2(5.26)$ \\
\hline Control & 38 & $5(13.16)$ & $3(7.89)$ & $4(10.53)$ & $12(31.58)$ \\
\hline $\mathrm{t} / \chi^{2}$ & & & & & 7.095 \\
\hline P-value & & & & & 0.007 \\
\hline
\end{tabular}

field. According to the number of cells with positive signal, a semi-quantitative scoring method was used assigning points for positivity and cell staining degree: 0 points for positive cell percentage of $<5 \%, 1$ point for positive cell percentage between 5 and $25 \%, 2$ points for positive cell percentage between 26 and 50\%, 3 points for positive cell percentage between 51 and $75 \%$ or 4 points for positive cell percentage $>75 \%$. Furthermore, scoring for staining included 0 points for no color, 1 point for pale yellow cells, 2 points for coarse granular brown cells, and 3 points for small block dark brown cells. The total sum of cell percentage plus cell staining degree points produced the total score. A total score $\leq 3$ indicated low-level expression, scores from 4 to 5 indicated moderate expression, and a score $\geq 6$ indicated a high expression.

Statistical analysis. Data were processed using SPSS 19.0 software (SPSS, Inc., Chicago, IL, USA). Measurement data were expressed as mean \pm standard deviation. The t-test was used for comparisons between the groups and within a group, and countable data were expressed as rates and processed using $\chi^{2}$ test, the treatment efficiency was evaluated by the rank-sum test. $\mathrm{P}<0.05$ was considered to be statistically significant.

\section{Results}

Comparison of the efficacy between two groups. The CR and PR of the observation group were 65.79 and $31.58 \%$, respectively. The CR and PR of the control group were 34.21 and $42.11 \%$, respectively. The total treatment efficacy of the observation group was significantly higher than that of the control group $(\mathrm{P}<0.05$, Table II).

Comparison of the treatment outcomes between two groups. The average healing time in the observation group was shorter than that in the control group. The number of treatments needed for those in the observation group was significantly smaller than the number of treatments needed for those in the control group. In addition, the apparent treatment satisfaction in the observation group was significantly higher than that in the control group $(\mathrm{P}<0.05$, Table III).

Comparison of the incidence of adverse reactions between two groups. The incidence of pain, redness, swelling and burning sensation in the observation group was significantly lower than that in the control group $(\mathrm{P}<0.05$, Table IV).

Comparison of recurrence rates between the two groups during the 12 months follow-ups. The recurrence rates in the observation group were 2.63 and $5.26 \%$ at 6 and 12 months after treatment, respectively. The recurrence rates in the control group were 13.15 and $34.21 \%$ at 6 and 12 months after treatment, respectively. While no significant difference in the recurrence rate was found at 6 months after treatment between the two groups $(\mathrm{P}>0.05)$, the recurrence rate 
Table V. Comparison of recurrence rate between the two groups (n, \%).

\begin{tabular}{lccc}
\hline Groups & $\mathrm{N}$ (cases) & $\begin{array}{c}\text { Recurrence } \\
\text { rate at } \\
6 \text { months } \\
\text { after treatment }\end{array}$ & $\begin{array}{c}\text { Recurrence } \\
\text { rate at } \\
12 \text { months } \\
\text { after treatment }\end{array}$ \\
\hline Observation & 38 & $1(2.63)$ & $2(5.26)$ \\
Control & 38 & $5(13.15)$ & $13(34.21)$ \\
$\mathrm{t} / \chi^{2}$ & & 1.627 & 8.308 \\
P-value & & 0.202 & 0.003 \\
\hline
\end{tabular}

of the observation group at 12 months after treatment was significantly lower than that of the control group at the same time $(\mathrm{P}<0.05$, Table $\mathrm{V})$.

Comparison of $C y P A, C y P B$ and $C D 147$ expression levels before and after treatment between two groups. The semi-quantitative scores of CyPA in the observation group before and after treatment were $6.16 \pm 0.62$ and $2.58 \pm 0.23$, respectively. The scores of $\mathrm{CyPB}$ before and after treatment were $6.06 \pm 0.43$ and $2.96 \pm 0.54$, respectively. The scores of CD147 before treatment and after treatment were $6.58 \pm 0.36$ and $2.18 \pm 0.15$, respectively. These results showed that treatment significantly decreased the expression of CyPA, CyPB and $\mathrm{CD} 147(\mathrm{P}<0.05)$ in the observation group. At the same time, the semi-quantitative scores of CyPA in the control group before and after treatment were $6.26 \pm 0.67$ and $3.98 \pm 0.43$, respectively. The scores of $\mathrm{CyPB}$ before and after treatment were $6.28 \pm 0.47$ and $3.96 \pm 0.57$, respectively. The scores of CD147 before and after treatment were $6.44 \pm 0.37$ and $3.18 \pm 0.25$, respectively, indicating that treatment significantly decreased the expression of CyPA, CyPB and CD147 in the control group as well $(\mathrm{P}<0.05)$. Of note, there was no significant difference in the expression of CyPA, CyPB and CD147 between the two groups before treatment $(\mathrm{P}>0.05)$. However, after treatment, the expression of CyPA, CyPB and CD147 in the observation group was significantly lower than those in the control group $(\mathrm{P}<0.05$, Figs. 1-3).

\section{Discussion}

Skin cancer is a common clinical malignant tumor, with basal cell carcinoma and squamous cell carcinoma being the two most common types that account for approximately $90 \%$ of all skin cancers (6). Skin cancer is usually aggressive and generates metastatic growth. Basal cell carcinoma, which accounts for the largest proportion of skin cancers, can initially grow within the epidermal basal cells with less metastasis, but with time the tumor nest can be extended to the dermal papilla (7). Squamous cell carcinoma, which accounts for the second largest proportion of skin cancers, originates from the epidermis or keratinocytes. Squamous cell carcinoma usually leads to different degrees of keratosis and lymphatic metastases (8). The possible mechanism of invasion and metastasis of skin cancer is explained by the ability of tumor cells to produce enzymes that can degrade extracellular matrix compo-



Figure 1. Semi-quantitative scores of CyPA before and after treatment.

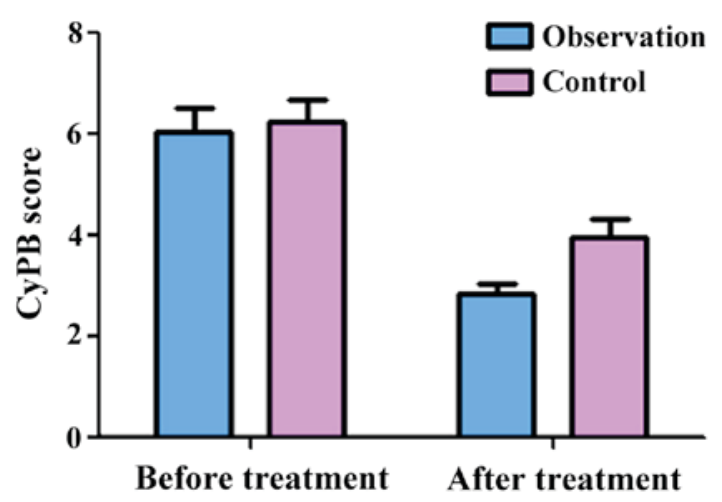

Figure 2. Semi-quantitative scores of CyPB before and after treatment.

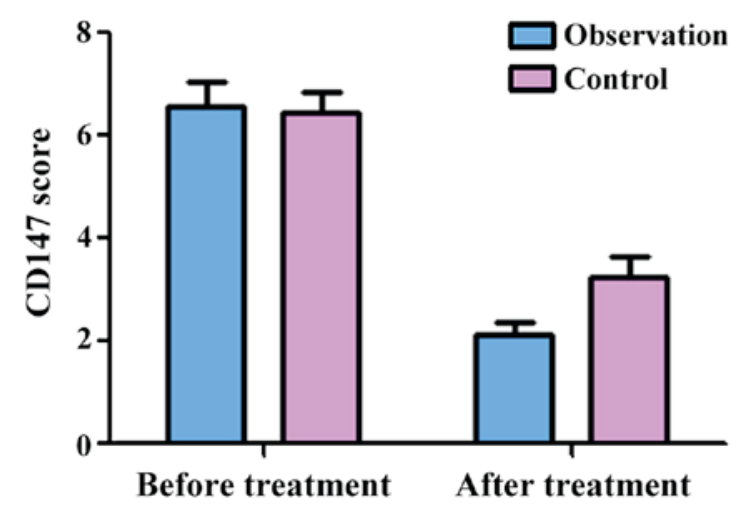

Figure 3. Semi-quantitative scores of CD147 before and after treatment.

nents facilitating the migration of tumor cells. At the same time, stromal or tumor cells are induced to produce adhesion molecules and cytokines, such as vascular endothelial growth factor to facilitate migration and neovascularization $(9,10)$. The etiology of skin cancer is not yet fully understood. It may be related to chemical stimulants, excessive sunlight exposure, long-term radiation exposure, or trauma (11).

Many treatment methods, including surgery, laser, cryotherapy, electrocautery and radiotherapy, can be used in the treatment of skin cancer, and surgical treatment is the gold standard $(1,4)$. The 5 -year cure rate of surgical treatment can reach $90 \%$ or even higher, but these treatment methods are usually followed by a persistent recurrence rate, that even if treated successfully, are likely to affect the patient's 
appearance $(12,13)$. Therefore, prevention of local recurrence and improvement of appearance have become hot research goals.

ALA-PDT, which combines a photosensitizer with a source light and oxygen can specifically destroy the lesion, thereby killing only tumor cells (14). As a precursor of hemoglobin, 5-ALA is a recently developed second-generation photosensitizer. 5-ALA can be absorbed by the rapidly proliferating cells and selectively accumulates in the cells, increasing the effectiveness of treatment (15). The results of our study showed the treatment of surgery and ALA-PDT was superior to the treatment with ALA-PDT alone, the efficacy rate was close to $100 \%$, the average wound healing time was significantly shorter, and the number of treatments needed significantly less. This is probably because surgical treatment can effectively open the tumor barrier, and remove most of the lesion to fully expose any hard to reach tumor cells, effectively increasing the depth of the tissue being treated. The surgical treatment facilitates the penetration of 5-ALA and increases the accumulation of 5-ALA in tumor cells, which in turn increase the production of protoporphyrin IX (PPIX) and hemoglobin (16). With the irradiation of light at $635 \mathrm{~nm}$ wavelengths, PPIX produces reactive oxygen species that specifically kill the tumor cells without bringing damage to the surrounding normal cells (17). Thus, the treatment effect of this combined method is stronger than that of ALA-PDT alone and the number of treatments needed is significantly less. In the case of facial tumors, surgical treatment combined with ALA-PDT is likely to achieve the best efficacy with the best cosmetic results ensuring higher appearance satisfaction (18).

Our results showed that no significant differences were found in the recurrence rates at 6 months after treatment between the two groups $(\mathrm{P}>0.05)$, while the recurrence rate of the observation group was significantly lower than that of the control group at 12 months after treatment $(\mathrm{P}<0.05)$. These data suggest that ALA-PDT can kill tumor cells by itself. Nevertheless, surgical treatment combined with ALA-PDT ensures eradication of tumor cells is more efficient in modulating the recurrence after longer periods of time.

CyPA and CyPB are the main members of the CyP family, CyPA is mainly located in the cytoplasm, while CyPB is mainly located in the endoplasmic reticulum, but can also be secreted outside of cells. Both CyPA and CyPB play important roles in cell-cell signaling transduction. CyPA and CyPB can block the dephosphorylation of T-cell activation factor, inhibit the transcription of IL-2 and other cytokines, and reduce the release of these cytokines to achieve their function of immunosuppression. CyPA and CyPB are highly expressed in pancreatic cancer cells, non-small cell lung cancer cells, tongue squamous cell carcinoma cells and many others (19). CD147, a member of the immunoglobulin superfamily, is widely distributed in the body. CD147 can stimulate fibroblasts to produce collagenase, which is involved in tumor infiltration and metastasis. CD147 is highly expressed in liver and lung cancer cells, lymphoma cells, skin squamous cell carcinomas and other cancer cells (20). The results of our study showed that the levels of CyPA, CyPB and CD147 in the two groups were significantly decreased after treatment and that the decrease in the observation group was significantly higher than that of the control group $(\mathrm{P}<0.05)$. It is possible that $\mathrm{CyPA}$ and $\mathrm{CyPB}$ were highly expressed in the tumor tissue before treatment, enhancing the expression of CD147 and its transportation to the cell membrane. CD147, in turn, promoted the secretion of CyPA and CyPB, a function for which it is known (21). Treatment with ALA-PDT can induce tumor cells to produce more TNF- $\alpha$, IL- $1 \beta$ and other pro-inflammatory cytokines, thereby activating $\mathrm{NF}-\kappa \beta$, promoting tumor cell apoptosis and necrosis, and effectively reducing the secretion of CyPA and CyPB. In addition, inhibiting the activation and angiogenesis of vascular endothelial cells, and blocking the interaction between CD147 and CyPA, and CyPB, will in turn prevent tumor invasion and metastasis, and reduce recurrence rates (21).

In conclusion, the treatment of surgery combined with ALA-PDT in skin cancer can fully take advantage of the strengths of each method. This approach cannot only increase the survival rate but can also improve the recurrence rate and even improve appearance. Further studies are required to promote this treatment method in the clinical practice.

\section{References}

1. LeSueur BW, DiCaudo DJ and Connolly SM: Axillary basal cell carcinoma. Dermatol Surg 29: 1105-1108, 2003.

2. Climstein M, Furness J, Hing W and Walsh J: Lifetime prevalence of non-melanoma and melanoma skin cancer in Australian recreational and competitive surfers. Photodermatol Photoimmunol Photomed 32: 207-213, 2016.

3. Leiter U, Eigentler T and Garbe C: Epidemiology of skin cancer. Adv Exp Med Biol 810: 120-140, 2014.

4. Lucena SR, Salazar N, Gracia-Cazaña T, Zamarrón A, González S, Juarranz A and Gilaberte Y: Combined treatments with photodynamic therapy for non-melanoma skin cancer. Int J Mol Sci 16: 25912-25933, 2015

5. Yoon HE, Oh SH, Kim SA, Yoon JH and Ahn SG: Pheophorbide amediated photodynamic therapy induces autophagy and apoptosis via the activation of MAPKs in human skin cancer cells. Oncol Rep 31: 137-144, 2014.

6. Abbas M and Kalia S: Trends in non-melanoma skin cancer (basal cell carcinoma and squamous cell carcinoma) in Canada: A descriptive analysis of available data. J Cutan Med Surg 20: 166-175, 2016.

7. Hussain AA, Themstrup L, Nürnberg BM and Jemec G: Adjunct use of optical coherence tomography increases the detection of recurrent basal cell carcinoma over clinical and dermoscopic examination alone. Photodiagn Photodyn Ther 14: 178-184, 2016.

8. Kauvar AN, Cronin T Jr, Roenigk R, Hruza, G and Bennett R: Consensus for nonmelanoma skin cancer treatment: Basal cell carcinoma, including a cost analysis of treatment methods. Dermatol Surg 41: 550-571, 2015.

9. Cho E and Grim JE: A (heat) shocking development: FBXW7 loss unleashes HSF1 to drive melanoma invasion and metastasis. Pigment Cell Melanoma Res 28: 643-644, 2015.

10. Campione E, Paternò EJ, Candi E, Falconi M, Costanza G, Diluvio L, Terrinoni A, Bianchi L and Orlandi A: The relevance of piroxicam for the prevention and treatment of nonmelanoma skin cancer and its precursors. Drug Des Devel Ther 9: 5843-5850, 2015.

11. Tang L, Yi XM, Chen J, Chen FJ, Lou W, Gao YL, Zhou J, Su LN, $\mathrm{Xu} \mathrm{X}, \mathrm{Lu} \mathrm{JQ}$, et al: Ubiquitin ligase UBE3C promotes melanoma progression by increasing epithelial-mesenchymal transition in melanoma cells. Oncotarget 7: 15738-15746, 2016.

12. Campos PM, Lopes Badra Bentley MV and Torchilin VP: Nanopreparations for skin cancer therapy. In: Nanobiomaterials in Cancer Therapy. Grumezescu AM (ed). Vol 7. 1st edition. Elsevier, New York, NY, pp1-28, 2016.

13. Dummer R: Precision medicine and skin cancer therapy: Dealing with a moving target. Curr Opin Oncol 26: 182-183, 2014.

14. Dixon AJ, Anderson SJ, Mazzurco JD and Steinman HK: Novel photodynamic therapy does not prevent new skin cancers randomized controlled trial. Dermatol Surg 40: 412-419, 2014. 
15. Na JI, Kim SY, Kim JH, Youn SW, Huh CH and Park KC: Indole-3-acetic acid: A potential new photosensitizer for photodynamic therapy of acne vulgaris. Lasers Surg Med 43: 200-205, 2011.

16. Valdes PA, Bekelis K, Harris BT, Wilson BC, Leblond F, Kim A, Simmons NE, Erkmen K, Paulsen KD and Roberts DW: 5-Aminolevulinic acid-induced protoporphyrin IX fluorescence in meningioma: Qualitative and quantitative measurements in vivo. Neurosurgery 10 (Suppl 1): 74-83, 2014.

17. Kanick SC, Davis SC, Zhao Y, Hasan T, Maytin EV, Pogue BW and Chapman MS: Dual-channel red/blue fluorescence dosimetry with broadband reflectance spectroscopic correction measures protoporphyrin IX production during photodynamic therapy of actinic keratosis. J Biomed Opt 19: 75002, 2014.

18. Sidoroff A and Thaler P: Taking treatment decisions in non-melanoma skin cancer - the place for topical photodynamic therapy (PDT). Photodiagn Photodyn Ther 7: 24-32, 2010.
19. Huang C, Sun Z, Sun Y, Chen X, Zhu X, Fan C, Liu B, Zhao Y and Zhang W: Association of increased ligand cyclophilin A and receptor CD147 with hypoxia, angiogenesis, metastasis and prognosis of tongue squamous cell carcinoma. Histopathology 60: 793-803, 2012

20. Fei F, Li X, Xu L, Li D, Zhang Z, Guo X, Yang H, Chen Z and Xing J: CD147-CD98hc complex contributes to poor prognosis of non-small cell lung cancer patients through promoting cell proliferation via the PI3K/Akt signaling pathway. Ann Surg Oncol 21: 4359-4368, 2014

21. Li L, Tang W, Wu X, Karnak D, Meng X, Thompson R, Hao X, Li Y, Qiao XT, Lin J, et al: HAb18G/CD147 promotes pSTAT3-mediated pancreatic cancer development via CD44s. Clin Cancer Res 19: 6703-6715, 2013. 